\title{
The impact of COVID-19 pandemic on the level of depression among health care workers: Cross-sectional Study
}

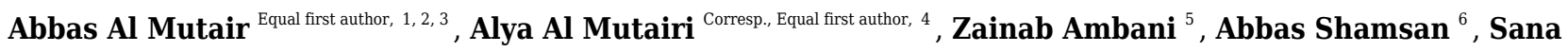 \\ AlMahmoud $^{7}$, Saad Alhumaid ${ }^{8}$ \\ ${ }^{1}$ Research Center, Almoosa Specialist Hospital, Alhasa, saudi Arabia \\ 2 Princess Norah University, Riyadh, Saudi Arabia \\ 3 University of Wollongong, Wollongong, Australia \\ 4 Department of Mathematics, Faculty of Science, Taibah University, MEDINA, SAUDI ARABIA \\ ${ }^{5}$ King Saud Ben Abdulaziz University for Health Sciences, Al Ahsa, SAUDI ARABIA \\ 6 Dr. Sulaiman Al Habib Medical Group, RIYADH, SAUDI ARABIA \\ 7 Imam Abdurrahman Bin Faisal University, RIYADH, SAUDI ARABIA \\ 8 Administration of Pharmaceutical Care, Ministry of Health, RIYADH, SAUDI ARABIA \\ Corresponding Author: Alya Al Mutairi \\ Email address: amutairi@taibahu.edu.sa
}

Background: The outbreak of the novel Corona Virus Infectious Disease 2019 (COVID-19) has spread rapidly to many countries leading to thousands of deaths globally. The burden of this pandemic has affected the physical and mental health of the frontline health care workers (HCWs) who are exposed to high risk of infection and psychological stressors. Aims: The aim is to measure the level of depression among healthcare workers in Saudi Arabia during COVID-19 pandemic to establish interventional strategies. Method: A descriptive cross-sectional study was used to conduct the current study. The data of this study was recruited between 15 June and 15 July 2020 from healthcare providers who work in both public and private healthcare sectors in Riyadh and Eastern province in Saudi Arabia utilizing a self-administered questionnaire. The study was approved by the Institutional Review Board at Dr Sulaiman Al Habib Medical Group (IRB Log No. RC20.06.88-2). Data were collected by using The Zung Self-Rating Depression Scale SDS. A total of 900 healthcare providers working in the healthcare setting during COVID-19 pandemic were invited to participate in the study. A total of 650 healthcare providers participated in the study by completing and submitting the survey. Results: Almost 30\% suffered from depression which can be divided into three categories; mild depression (26.2\%), moderate/major (2.5\%) and severe/extreme $(0.8 \%)$. The finding shows that the level of depression among respondents at the age range of 31-40 years old was significantly higher than the level of depression among respondents with the age above 50 years old. Non-Saudi healthcare workers experienced more depression than Saudi workers. 
It also shows how nurses suffered from depression compared to their physician colleagues. Those who did not suffer from sleeping disorder perceived more depression as compared to those who are having sleeping disorder. Conclusion: It is recommended that health care facilities should implement strategies to reduce the prevalence of mental health problems among healthcare providers and eventually it will improve their performance in provision of safe and high-quality care for patients. 


\title{
The Impact of COVID-19 Pandemic on the Level of Depression among Health Care Workers: Cross-sectional Study
}

\author{
ABBAS AL MUTAIR ${ }^{1,2,3}$, ALYA AL MUTAIRI ${ }^{4 *}$, ZAINAB AMBANI ${ }^{5}$, ABBAS SHAMSAN $^{6}$, SANA \\ ALMAHMOUD $^{7,8}$ AND SAAD ALHUMAID $^{9}$ \\ 1. Research Center, Almoosa Specialist Hospital, Al-ahsa, Saudi Arabia, \\ 2. Princess Norah University, Riyadh, Saudi Arabia. \\ 3. University of Wollongong, Australia; abbas4080@hotmail.com \\ 4. Department of Mathematics, Faculty of Science, Taibah University, Medina, Saudi Arabia. \\ *Correspondence: amutairi@taibahu.edu.sa \\ 5. King Saud Ben Abdulaziz University for Health Sciences, Al Ahsa, Eastern Province, Saudi Arabia; \\ zaianb.ambani5@gmail.com \\ 6. Research Center, Dr. Sulaiman Al Habib Medical Group, Riyadh, Saudi Arabia; \\ abbas.shamsan@drsulaimanalhabib.com \\ 7. Research Center, Almoosa Specialist Hospital, Al-ahsa, Saudi Arabia \\ 8. Imam Abdurrahman Bin Faisal University, Saudi Arabia; saalmahmoud@iau.edu.sa \\ 9. Administration of Pharmaceutical Care, Ministry of Health, Riyadh, Saudi Arabia; \\ saalhumaid@moh.gov.sa
}

Corresponding Author:

ALYA AL MUTAIRI

Department of Mathematics, Faculty of Science, Taibah University, Medina, Saudi Arabia. *Correspondence: Email address: amutairi@taibahu.edu.sa

\section{Abstract}

Background: The outbreak of the novel Corona Virus Infectious Disease 2019 (COVID-19) has spread rapidly to many countries leading to thousands of deaths globally. The burden of this pandemic has affected the physical and mental health of the frontline health care workers (HCWs) who are exposed to high risk of infection and psychological stressors.

Aims: The aim is to measure the level of depression among healthcare workers in Saudi Arabia during COVID-19 pandemic to establish interventional strategies.

Method: A descriptive cross-sectional study was used to conduct the current study. The data of this study was recruited between 15 June and 15 July 2020 from healthcare providers who work in both public and private healthcare sectors in Riyadh and Eastern province in Saudi Arabia utilizing a self-administered questionnaire. The study was approved by the Institutional Review Board at Dr Sulaiman Al Habib Medical Group (IRB Log No. RC20.06.88-2). Data were collected by using The Zung Self-Rating Depression Scale SDS. A total of 900 healthcare providers working in the healthcare setting during COVID-19 pandemic were invited to participate in the study. A total of 650 healthcare providers participated in the study by completing and submitting the survey. 
41 Results: Almost 30\% suffered from depression which can be divided into three categories; mild 42 depression (26.2\%), moderate/major (2.5\%) and severe/extreme (0.8\%). The finding shows that the level 43 of depression among respondents at the age range of 31-40 years old was significantly higher than the 44 level of depression among respondents with the age above 50 years old. Non-Saudi healthcare workers 45 experienced more depression than Saudi workers. It also shows how nurses suffered from depression 46 compared to their physician colleagues. Those who did not suffer from sleeping disorder perceived more 47 depression as compared to those who are having sleeping disorder.

48 Conclusion: It is recommended that health care facilities should implement strategies to reduce the 49 prevalence of mental health problems among healthcare providers and eventually it will improve their 50 performance in provision of safe and high-quality care for patients.

52 Keywords: COVID-19; Depression; mental health; sleeping disorder; health care workers; health care 53 facilities; Saudi Arabia

\section{Background}

56 Mental health is one of the important issues that receives attention from the World Health Organization 57 (WHO) as it was included in the Sustainable Developmental Goals for 2030. Over the last decade, mental health problems increased by $13 \%$ worldwide [1]. The most common mental health conditions are depression and anxiety which cost around US \$ 1 trillion every year from global economy [2]. Mental health problems have a serious impact on all aspects of life such as interacting with others, family members, co-workers and even with patients. Despite that, the global median expenditure is less than 2\% of the governmental health expenditure. The spread of COVID-19 pandemic caused huge stress on general population, however, the case was worse for healthcare workers due to their close interaction with COVID-19 patients [2].

Frontline healthcare workers (HCWs) are dealing with suspected and positive cases of COVID-19 patients on daily basis which puts them at high risk of infection and death [3]. Such exposure has a substantial impact on their mental health $[4,5,6]$. One of the most common mental health problems is depression. Depression can cause significant physical, emotional and behavioral problems [7, 8, 9]. More than two thirds of healthcare workers serving in Turkey experienced high level of depression [10]. Being a female, a nurse, and having a history of psychiatric illness were identified as risk factors for mental health problems [10]. Many mental conditions can be treated or minimized by adopting some changes in 72 the workplace, at home or in the community. However, evidence-based decisions need to be taken prior to 73 implementing any strategy. Therefore, studies in this field are needed in order to examine the nature of 74 the mental issues and how to reduce it. Since the spread of COVID-19 pandemic, researchers have 
75 conducted numerous studies to assess the impact of the COVID-19 on the physical and mental wellbeing 76 of individuals. The target population varies according to the purpose of the study. Some studies examined 77 the impact of the disease on COVID-19 patients [11]; some focused on general populations [12, 13, 14], 78 and some studies were specific to frontline healthcare workers $[15,16,17]$.

79 The psychological burden of health care workers had received attention in research publications. 80 Numerous studies reported consistent results of health workers' stress, anxiety, and depressive symptoms 81 due to COVID-19 [18]. Healthcare workers experience more psychological symptoms than non82 healthcare workers. These symptoms include insomnia, anxiety, depression and obsessive -compulsive 83 [14]. Moreover, studies also showed that HCWs experience emotional exhaustion, burnout, stress, and 84 suicide [19]. A study conducted in the Second Xiangya Hospital in China where 13 medical staff were 85 interviewed to assess the mental health care during COVID-19 pandemic. Results showed how nurses 86 presented signs of psychological distress, yet they refused receiving any psychological help considering 87 that not a problem. They focused on the need of more uninterrupted rest and enough protective equipment 88 [15]. When frontline HCWs in Wuhan itself where surveyed, findings showed that HCWs were under moderate to severe stress and many reported elevated anxiety and depression [15]. Italian healthcare providers also demonstrated high anxiety and depression levels during COVID-19 pandemic [19]. This was a cross-sectional study which surveyed healthcare providers including doctors and nurses utilizing quality of life and health-related Visual Analogue Scales, State-Trait Anxiety Inventory, Beck Depression Inventory and PTSD Checklist [19]. Findings of the current Italian study found that female healthcare providers were found to have higher levels of depression symptoms [19]. According to previous published literature, working in hospitals in general was associated with depression symptoms among healthcare providers [20]. Depression symptoms were reported more during COVID-19 pandemic among frontline healthcare providers [20]. In addition, high burnout level among Saudi and non-Saudi healthcare providers, it is crucial to deal with the issue for public health in Saudi Arabia and UAE [21]. Healthcare providers are more vulnerable to depression due to high turnover, heavy workload and fear of infections due to contagious nature of disease such as COVID-19 [22]. As this pandemic continues to spread, its physical, emotional and mental impact on Saudi population and globally continues contributing to increase depression and emotional level [23]. Working in healthcare facilities have high amounts of psychological distress in doctors, nurses, and other health-care providers [24]. Results of previously conducted study in Saudi Arabia showed that healthcare workers had high level of depression and anxiety indicating greater psychological distress. In the study female healthcare workers had more depression than their male colleagues, physicians had lesser psychological distress compared to other healthcare providers and Non-Saudi had higher psychological distress than Saudi professionals [24]. COVID-19 has spread all over the world affecting many individuals', especially 
109 healthcare providers. Previous studies have shown that clinicians in China suffered mental health 110 problems from COVID-19 pandemic [25]. In one of the studied which was conducted in 34 Chinese 111 hospital surveyed 1,257 healthcare workers found that frontline healthcare workers and female nurses had 112 higher risk of developing mental health issues and needed psychological support or medical interventions.

113 Reports have also revealed that healthcare providers suffered from mental health problems during Sever 114 Acute Respiratory Syndrome SARS [26, 27, 28, 29, 30, 31, 32]. Similarly, the prevalence of mental 115 illnesses during Middle East Respiratory Syndrome (MERS) epidemic was high among healthcare 116 providers in Saudi Arabia [33]. Previous studies showed high prevalence of COVID-19 cases in Saudi 117 Arabia ranging from mild to severe characteristics [34, 35]. Furthermore, during COVID-19 pandemic 118 studies have also shown that anxiety among healthcare providers in Saudi Arabia was high due to looking 119 after COVID-19 patients [36]. Due to the lack of social support and communication, maladaptive coping, 120 and lack of training on how to deal with such pandemic were identified as risk factors for developing 121 psychological problems such as depression, anxiety, insomnia and distress, especially to those healthcare 122 workers who interact directly with suspected and confirmed COVID-19 patients [15]. Individuals with 123 pre-existing psychiatric disorders reported deterioration of their mental health conditions due to COVID12419 [3]. Level of social support for medical staff is a significant predictor for self- efficacy and sleep 125 quality and negatively associated with anxiety and stress [37]. The ongoing COVID-19 pandemic along 126 with the measures to control it can lead to mental issues such depression. While the cases of COVID-19 127 attending healthcare centers and hospitals has been rapidly increasing along with extended hours of work 128 can place huge mental pressure on healthcare providers. Given the cultural variations in responding to 129 pandemics, it is highly important to investigate how healthcare providers in Saudi Arabia react 130 emotionally and mental to COVID-19. This may help the government to initiate supportive programs to 131 decrease and control healthcare providers depression and anxiety which may contribute to improve their 132 psychological well-being. This study intends to investigate the level of depression among healthcare 133 providers in Saudi Arabia

134

135 Methods

136 Study design

137 A descriptive cross-sectional non-interventional design was used for the purpose of this study. 138 Anonymous self-administered, paper and pencil survey was used to collect data from the targeted setting. 139 The researchers obtained the ethical approval to conduct the study from the Institutional Review Board at 140 Dr. Sulaiman Al Habib Medical Group (IRB Log No. RC20.06.88-2). Participation in the study was 141 voluntary and participants were ensured that information gathered for the study would be kept 142 confidential and will be used for the study purposes only and consent letter was requested for this study as 
143 well. Level of depression is the dependent variable in the current study which will be measured by the

144 Zung Self-Rating Depression Scale SDS. The soci-demographic profile of the participants is the

145 independent variable. The initial sample size was estimated using $\mathrm{G}^{*}$ Power3 and based on multiple linear

146 regression using the independent two-tailed t-test, confidence level of $95 \%$, margin rate of error at $5 \%$ and

147 power of $80.0 \%$, medium effect size of .30 (determined based on the review of current literature), and a

$14810 \%$ increase to address the non-response rate, the minimum required sample size for this study was 353

149 subjects. A total of 900 surveys were distributed among healthcare providers, 650 returned back the 150 completed surveys giving a response rate of $72 \%$.

151 Saudi and non-Saudi healthcare providers were eligible to be a participant in this study if all of the 152 following criteria are met: (1) is 18 years of age or older (2) works in inpatient or outpatient healthcare 153 setting (3) is responsible for providing direct bedside patient care, and (4) has spent at least three months 154 in current healthcare unit. Healthcare providers who work in private and public healthcare sectors in 155 Riyadh, Saudi Arabia were invited to participate in the study by completing and returning back the 156 questionnaire. Data collection continued for two weeks between 2 to 15 April, 2020.

157

158

\section{Data collection instrument}

159 The Zung Self-Rating Depression Scale SDS was used to collect data from the participants. It consists of 160 20 items which has been widely used as a screening tool for depression in multiple populations. The SDS

161

162

163

164

165

166

167

168

169

170

171

172

173

174

175 statements are framed in a positive and negative pattern with 4-Likert point scale ranging from 1 a little of the time, 2 some of the time, 3 good part of the time and 4 most of the time. The scale score may range from 20 to 80, scores from 20 to 49 indicating no depression, 50 to 69 indicating depression and scores from 70 to 80 indicting severe depression [38]. The scale has shown good validity and acceptable reliability for clinical and research purposes with alpha value 0.84 [38]. Additional part to the questionnaire about the socio-demographic characteristics of the respondents was added in the current study. This included age, gender, marital status, economic status, nationality, profession, working area, years of working experience and type of healthcare facility.

\section{Data analysis}

Frequencies and percentages for nominal/ordinal level variables was employed. Chi-square analysis has been conducted to determine the association between demographic profile and depression. Unpaired Independent t-test and one-way ANOVA analysis to compare healthcare providers' level of depression and predict the factors of depression among the study populations. P-value of $\leq 0.05$ were accepted as the significance level for all inferential statistical tests. 


\section{Results}

\section{Demographic profile analysis}

178 Demographic profile has been analyzed and the findings are presented in the Table 1. Several 179 demographic profiles have been chosen namely the type of health care facility, age, gender, nationality, 180 health specialty, hospital department, years of experience, sleeping disorder before covid-19 and mental 181 disorder. In terms of type of health care facilities, $444(68.7 \%)$ of participants work in private facilities 182 while only $202(31.3 \%)$ work in governmental hospitals. By looking at the age, about $177(46.6 \%)$ of the 183 respondents were at the age range 31-40 years old, followed by 123 (32.4\%) were 20-30 years old, 63 $184(16.6 \%)$ were 41-50 years old and a few number of workers $17(4.5 \%)$ were above 50 years old. The 185 percentage of females $(n=475,74 \%)$ was larger than the percentage of males $(n=167,26 \%)$. Almost three 186 quarters of the respondents 450 (70.4\%) were non-Saudis while 189 (26\%) were Saudis. By studying at 187 the health specialty, $323(50.2 \%)$ of the respondents were nurses, $114(17.7 \%)$ were physicians, and 207 188 (32.1\%) were from other specialties. Approximately $243(38.3 \%)$ of the respondents work in Intensive 189 Care Unit (ICU), followed by 206 (32.4\%) work in other departments, 134 (21.1\%) work in wards and 52 190 (8.2\%) work in Emergency department. By studying at the year of experience, $280(44.3 \%)$ have 1 to 5 191 years of work experience, followed by $177(28 \%)$ with 6 to 10 years of work experience and $175(27.7 \%)$ with over 11 years of work experience. Respondents were asked about sleeping disorder before COVID19, about 532 (86.9\%) did not have sleeping disorder while 80 (13.1\%) had sleeping disorder before COVID-19. Lastly, in terms of mental disorder, about 592 (96.7\%) did not have mental disorder, while 20 (3.3\%) had mental disorder.

196

\section{Level of depression}

The Level of depression has been divided into four categories as shown in Table 2, which they are normal, mild depression, moderate/major and severe/extreme. Almost over three quarters of respondents did not suffer from depression which comprised 459 (70.6\%). Furthermore, the findings revealed less than $30 \%$ suffer from depression which falls into three categories; mild depression 170 (26.2\%), moderate/major $16(2.5 \%)$ and severe/extreme $5(0.8 \%)$ as shown in Figure 1.

203

\section{The association between demographic profile and depression}

205

206

207

208
Chi-square analysis has been conducted to determine the association between demographic profile and depression as shown in Table 3. Several demographic profiles have been selected which are health care facilities, age, gender, nationality, health specialty, work area, years of experience, sleeping disorder before COVID-19 and mental disorder. The findings have presented in Table 4 further revealed age $[X 2=12.89, d f=6, p<0.05]$, nationality $[X 2=8.321, d f=2, p<0.05]$, health specialty $[X 2=9.54, d f=4, p<0.05]$ 
210 and sleeping disorder before COVID-19 [X2=12.127, $d f=2, p<0.05]$ have significant association with 211 depression. However, healthcare facility $[X 2=2.377, d f=2, p>0.05]$, gender $[X 2=3.683, d f=2, p>0.05]$, 212 hospital department (work area) $[X 2=10.216, d f=6, p>0.05]$, years of experience $[X 2=7.134, d f=4$, $213 p>0.05]$ and mental disorder $[X 2=5.919, d f=2, p>0.05]$ insignificantly influenced depression. The findings 214 showed that among respondents at the age range of 31-40 years old, the level of depression was 215 [46.6 \pm 39.59$]$ significantly higher than respondents with the age above 50 years old [4.5 \pm 32.37$]$. In terms 216 of nationality, non-Saudi [70.4 \pm 40.68 ] suffered from depression compared to Saudi [29.6 \pm 40.08$]$. Other 217 healthcare providers' specialties [32.1 \pm 41.70$]$ were more depressed than physicians [17.7 \pm 39.70$]$. Those 218 who did not suffer from sleeping disorder [86.9 \pm 39.93$]$ perceived depression compared to those who are 219 having sleeping disorder [13.1 \pm 41.20$]$.

\section{Discussion}

221 Depression and anxiety are common and cited problems that generally encounter individuals and more 222 specifically frontline healthcare workers [21, 27]. Our study aimed to measure the level of depression 223 among healthcare workers in Saudi Arabia during COVID-19 pandemic and to measure the level of 224 depression based on demographic profile. Several limitations should be taken into account while 225 interpreting the results of the current study. A cross-sectional design was used to recruit healthcare 226 providers from Riyadh capital city in Saudi Arabia, so caution must be practiced while generalizing its

227

228

229

230

231

232

233

234

235

236

237

238

239

240

241 results to all healthcare providers in the county. Additionally, current findings rely heavily on subjective perception of healthcare providers rather than objective methods or clinical diagnosis and therefore further studies on prevalence of depression among healthcare providers should be conducted subjectively during and after the pandemic.

In our study, the prevalence of depression among healthcare workers was $29.5 \%$ which is close to its percentage in Turkey (31.8\%) [27]. Since the spread of COVID-19 in Wuhan and Hubei in China, psychological distress was reported in $70 \%$ of healthcare workers [9]. In Iran, the prevalence of depression among HCWs was reported as 30\% [28]. The classifications of depression levels in this study is similar to other studies that found the higher percentage of those with depression symptoms had mild depression, followed by those with moderate depression, and the least percentage was the HCWs who suffer from severe depression [17]. For instance, in other studies, healthcare providers from Saudi Arabia and Egypt reported high level of depression, anxiety and stress during COVID-19 pandemic [37]. Young female healthcare providers had scored higher on depression and stress scales especially those who were recruited from Egypt. Researchers have suggested psychological support interventions programs targeting healthcare providers. 
242 It is expected that the percentage in China is higher than in other countries due to lack of information on 243 how to deal with this new virus at the beginning of its onset. Upon the spread of the infection to other 244 countries, some information about the virus and precautions became available which minimized the 245 uncertainty. The fear of being infected by COVID-19 is the core cause of anxiety and other mental health 246 problems. Some of the reported risk factors for that are spending long hours in hospitals with COVID-19 247 patients, suboptimal hand cleanliness, and lack of protective equipment $[15,34]$. The findings show that 248 the level of depression was significantly high for HCWs whom age is between 31 to 40 years respondents 249 compared to those who are above 50 years old. This could be related to social responsibilities for this age 250 group who are usually married, have children or family members who are at old age and considered as the 251 most vulnerable group. Nationality variable was not studied previously in similar studies. The results 252 show a significantly higher level of depression among non-Saudi HCWs as compared to Saudi HCWs. In 253 addition to the stress and anxiety associated with working in hospitals with COVID-19 patients, non254 Saudi encounter more stressors such as not being able to visit their families, return to their home 255 countries, the fear and worries of getting infected, facing death alone, or having a family member dying 256 while they are away from home. These tragedies have happened in reality and they are leading to higher 257 levels of anxiety and depression to non-Saudi HCWs. Consistent with other studies, nurses are 258 experiencing more depression than other HCW [15]. Although physicians are dealing with COVID-19 259

260

261

262

263

264

265

266

267

268

269

270

271

272 effectively and safely. Some of the suggested interventions are:

273 1. Develop strategies in the workplace to optimize the mental health of HCWs such as creating places to 274 exercise, mindfulness, meditation, and places for rest and sleep especially for those who work long 275 hours. 
276 2. Ensure availability of Personal Protective Equipment (PPE) to all HCWs. This will reduce the worries

277 of being infected and will increase the sense of security and safety

278 3. Monitor strict adherence to all safety precautions while dealing with patients and colleagues at $279 \quad$ workplace.

280 4. Modify the HCWs' work schedule to have shorter shifts to allow workers to rest and socialization $281 \quad$ with their families.

282 5. Allow HCWs to use technology and telehealth when there is no need for direct contact with patients 283 to minimize the exposure to infection.

6. Encourage HCWs to seek help and consultation whenever they start feeling any anxiety, depression, insomnia or other mental health problems. This will reduce the complication of cases and help them receiving the appropriate treatment earlier.

287

288

289

290

291

292

293

294

295

296

297

298

299

300

301

302

303

304

305

306

308

309

These interventions may improve the mental health of the HCWs and prevent further complications if they were addressed early. The current study findings should be interpreted taking into full considerations its limitations. The study sample is composed represent private facilities more than government hospital (68.7\% Vs. $31.3 \%$ respectively), and the percentage of non-Saudis were higher than Saudis (70.4\% Vs. $29.6 \%$ respectively). This may limit the generalization of results.

\section{Conclusion}

Overall, level of depression has been divided into four levels. The findings revealed less than $30 \%$ suffer from depression which fall into three categories; mild depression 170 (26.2\%), moderate/major 16 (2.5\%) and severe/extreme $5(0.8 \%)$. Several demographic profiles have been selected which are healthcare facilities, age, gender, nationality, health specialty, hospital department, years of experience, sleeping disorder before Covid-19 and mental disorder. The findings further revealed age, nationality, health specialty and sleeping disorder before Covid-19 have significant association with depression. However, healthcare facilities, gender, hospital department, years of experience and mental disorder insignificantly influenced depression. Those who did not suffer from sleeping disorder perceived more depression as compared to those who are having sleeping disorder. It is recommended that health care facilities develop and implement some strategies to reduce the prevalence of mental health problems especially depression and insomnia. The impact of these strategies may improve the mental health of HCWs and eventually improve their performance in provision of safe and high quality care for patients.

\section{List of Abbreviations}

COVID-19: Coronavirus disease 201; SARS-CoV-2: Severe acute respiratory syndrome coronavirus 2; WHO: World Health Organization; IRB: Institutional Review Board; HCWs: Health care works; SPSS: 
310 Statistical Package for the Social Sciences; SD: Standard deviation; IQR: Interquartile range; SDS: Self-

311 Rating Depression Scale.

312

313 Ethics approval and consent to participate

314 This study was approved by the Institutional Review Board (IRB Log No. RC20.06.88-2).

315

316 Consent for publication

317 Participation in the study was voluntary and participants were ensured that information gathered for the

318 study would be kept confidential and will be used for the study purposes only.

319

320

\section{Availability of data and materials}

321 Not applicable

322

323

\section{Competing interests}

324

Authors declare no conflict of interest in preparing this article

325

326

\section{Funding}

327

Not applicable

328

329

330

\section{Authors' contributions}

331

ABBAS AL MUTAIR: Conceptualization, writing-original draft preparation, supervision, ethical

332 approval; ALYA AL MUTAIRI: Formal analysis, Data analysis, Interpretations, supervision; ZAINAB AMBANI: Methodology, writing-review and editing; ABBAS SHAMSAN: Resources, data curation, writing-original draft preparation; SANA ALMAHMOUD: Resources, data curation, writing-review and editing; SAAD ALHUMAID: Resources, data curation, writing-original draft preparation.

335

\section{Acknowledgements}

337 This The authors declare no conflict of interest in preparing this article, authors thank the referee for 338 constructive comments.

\section{References}

340 1. World Health Organization (WHO). WHO Coronavirus Disease (COVID-19) Dashboard. Retrieved on January $17^{\text {th }}, 2021$ from https://covid19.who.int/ 
342 2. World Health Organization (WHO). Maintaining essential health services during the COVID-19

343

344

345

346

347

348

349

350

351

352

353

354

355

356

357

358

359

360

361

362

363

364

365

366

367

368

369

370

371

372

373

374 outbreak. Retrieved on Nov. 14, 2020 from https:/www.who.int/emergencies/diseases/novelcoronavirus-2019/related-health-issues

3. Chirico, F., \& Magnavita, N. (2020). COVID-19 infection in Italy: An occupational injury. SAMJ: South African Medical Journal, 110(6), 0-0.

4. Yörük, S., \& Güler, D. (2020). The relationship between psychological resilience, burnout, stress, and sociodemographic factors with depression in nurses and midwives during the COVID-19 pandemic: A cross-sectional study in Turkey. Perspectives in Psychiatric Care.

5. Bohlken, J., Schömig, F., Lemke, M. R., Pumberger, M., \& Riedel-Heller, S. G. (2020). COVID-19Pandemie: Belastungen des medizinischen Personals: Ein kurzer aktueller review. Psychiatrische Praxis, 47(4), 190.

6. Ran, L., Chen, X., Wang, Y., Wu, W., Zhang, L., \& Tan, X. (2020). Risk factors of healthcare workers with coronavirus disease 2019: a retrospective cohort study in a designated hospital of Wuhan in China. Clinical Infectious Diseases, 71(16), 2218-2221.

7. González-Sanguino, C., Ausín, B., Castellanos, M. A., \& Muñoz, M. (2020). Mental health consequences of the COVID-19 outbreak in Spain. A longitudinal study of the alarm situation and return to the new normality. Progress in Neuro-Psychopharmacology and Biological Psychiatry, 110219.

8. Zhou, J., Liu, L., Xue, P., Yang, X., \& Tang, X. (2020). Mental health response to the COVID-19 outbreak in China. American Journal of Psychiatry, 177(7), 574-575.

9. Zhou, J., Zogan, H., Yang, S., Jameel, S., Xu, G., \& Chen, F. (2020). Detecting community depression dynamics due to covid-19 pandemic in australia. arXiv preprint arXiv:2007.02325.

10. Şahin, M. K., Aker, S., Şahin, G., \& Karabekiroğlu, A. (2020). Prevalence of depression, anxiety, distress and insomnia and related factors in healthcare workers during COVID-19 pandemic in Turkey. Journal of Community Health, 45(6), 1168-1177.

11. Bo, H. X., Li, W., Yang, Y., Wang, Y., Zhang, Q., Cheung, T., ... \& Xiang, Y. T. (2020). Posttraumatic stress symptoms and attitude toward crisis mental health services among clinically stable patients with COVID-19 in China. Psychological medicine, 1-2.

12. Fullana, M. A., Hidalgo-Mazzei, D., Vieta, E., \& Radua, J. (2020). Coping behaviors associated with decreased anxiety and depressive symptoms during the COVID-19 pandemic and lockdown. Journal of Affective Disorders, 275, 80-81.

13. Gao, J., Zheng, P., Jia, Y., Chen, H., Mao, Y., Chen, S., ... \& Dai, J. (2020). Mental health problems and social media exposure during COVID-19 outbreak. Plos one, 15(4), e0231924. 
375

376

377

378

379

380

381

382

383

384

385

386

387

388

389

390

391

392

393

394

395

396

397

398

399

400

401

402

403

404

405

406

407

408

14. Zhang, W. R., Wang, K., Yin, L., Zhao, W. F., Xue, Q., Peng, M., ... \& Wang, H. X. (2020). Mental health and psychosocial problems of medical health workers during the COVID-19 epidemic in China. Psychotherapy and psychosomatics, 89(4), 242-250.

15. Chen, Q., Liang, M., Li, Y., Guo, J., Fei, D., Wang, L., ... \& Zhang, Z. (2020). Mental health care for medical staff in China during the COVID-19 outbreak. The Lancet Psychiatry, 7(4), e15-e16.

16. Du, J., Dong, L., Wang, T., Yuan, C., Fu, R., Zhang, L., ... \& Li, X. (2020). Psychological symptoms among frontline healthcare workers during COVID-19 outbreak in Wuhan. General hospital psychiatry.

17. Lai, J., Ma, S., Wang, Y., Cai, Z., Hu, J., Wei, N., ... \& Hu, S. (2020). Factors associated with mental health outcomes among health care workers exposed to coronavirus disease 2019. JAMA network open, 3(3), e203976-e203976.

18. Shreffler, J., Petrey, J., \& Huecker, M. (2020). The impact of COVID-19 on healthcare worker wellness: A scoping review. Western Journal of Emergency Medicine, 21(5), 1059.

19. Di Tella, M., Romeo, A., Benfante, A., \& Castelli, L. (2020). Mental health of healthcare workers during the COVID-19 pandemic in Italy. Journal of evaluation in clinical practice, 26(6), 1583-1587.

20. Santarone, K., McKenney, M., \& Elkbuli, A. (2020). Preserving mental health and resilience in frontline healthcare workers during COVID-19. The American journal of emergency medicine, 38(7), 1530-1531.

21. Al-Omari, A., Al Mutair, A., Shamsan, A., \& Al Mutairi, A. (2020). Predicting burnout factors among healthcare providers at private hospitals in Saudi Arabia and United Arab Emirates: A crosssectional study. Applied Sciences, 10(1), 157.

22. Al Mutair, A., Alhajji, M., \& Shamsan, A. (2021). Emotional Wellbeing in Saudi Arabia During the COVID-19 Pandemic: A National Survey. Risk Management and Healthcare Policy, 14, 1065.

23. Al Mutair, A., Al Obaidan, F., Al-Muhaini, M., Al Salman, K., \& Al Mosajen, S. (2017). Crosssectional study of the overall emotional functioning of health-care providers in Saudi. Saudi Critical Care Journal, 1(3), 80.

24. Huang, C., Wang, Y., Li, X., Ren, L., Zhao, J., Hu, Y., ... \& Cao, B. (2020). Clinical features of patients infected with 2019 novel coronavirus in Wuhan, China. The lancet, 395(10223), 497-506.

25. Lee, A. M., Wong, J. G., McAlonan, G. M., Cheung, V., Cheung, C., Sham, P. C., \&amp; Chua, S. E. (2007). Stress and psychological distress among SARS survivors 1 year after the outbreak. The Canadian Journal of Psychiatry, 52(4), 233-240.

26. McAlonan, G. M., Lee, A.M., Cheung, V., Cheung, C., Tsang, K.W., Sham, P.C., Chua, S.E., Wong, 14 J.G., 2007. Immediate and sustained psychological impact of an emerging infectious disease 15 outbreak on health care workers. Can J Psychiatry 52, 241-247. doi: 16

PeerJ reviewing PDF | (2021:02:58059:3:0:NEW 13 Apr 2021) 
409

410

411

412

413

414

415

416

417

418

419

420

421

422

423

424

425

426

427

428

429

430

431

432

433

434

435

436

437

438

439

440

\subsection{7/070674370705200406}

27. Lai, J., Ma, S., Wang, Y., Cai, Z., Hu, J., Wei, N., ... \& Hu, S. (2020). Factors associated with mental health outcomes among health care workers exposed to coronavirus disease 2019. JAMA network open, 3(3), e203976-e203976.

28. Al Mutair, A., \& Ambani, Z. (2020). Narrative review of Middle East respiratory syndrome coronavirus (MERS-CoV) infection: updates and implications for practice. Journal of International Medical Research, 48(1), 0300060519858030.

29. Al Mutair, A., Alhumaid, S., Alhuqbani, W. N., Zaidi, A. R. Z., Alkoraisi, S., Al-Subaie, M. F., ... \& Al-Omari, A. (2020). Clinical, epidemiological, and laboratory characteristics of mild-to-moderate COVID-19 patients in Saudi Arabia: an observational cohort study. European Journal of Medical Research, 25(1), 1-8.

30. Chirico, F., \& Nucera, G. (2020). Tribute to healthcare operators threatened by COVID-19 pandemic. J Health Soc Sci, 5(2), 165-168.

31. Chirico, F., Nucera, G., \& Magnavita, N. (2021). Protecting the mental health of healthcare workers during the COVID-19 emergency. BJPsych International, 18(1).

32. Pappa, S., Ntella, V., Giannakas, T., Giannakoulis, V. G., Papoutsi, E., \& Katsaounou, P. (2020). Prevalence of depression, anxiety, and insomnia among healthcare workers during the COVID-19 pandemic: A systematic review and meta-analysis. Brain, behavior, and immunity.

33. Al-Omari, A., Alhuqbani, W. N., Zaidi, A. R. Z., Al-Subaie, M. F., AlHindi, A. M., Abogosh, A. K., ... \& Al Mutair, A. (2020). Clinical characteristics of non-intensive care unit COVID-19 patients in Saudi Arabia: a descriptive cross-sectional study. Journal of infection and public health, 13(11), 1639-1644.

34. Alenazi, T. H., BinDhim, N. F., Alenazi, M. H., Tamim, H., Almagrabi, R. S., Aljohani, S. M., ... \& Alqahtani, S. A. (2020). Prevalence and predictors of anxiety among healthcare workers in Saudi Arabia during the COVID-19 pandemic. Journal of infection and public health, 13(11), 1645-1651.

35. Al Mutair, A., Al Mutairi, A., Chagla, H., Alawam, K., Alsalman, K., \& Ali, A. (2020). Examining and adapting the psychometric properties of the Maslach burnout inventory-health services survey (MBI-HSS) among healthcare professionals. Applied Sciences, 10(5), 1890.

36. Xiao, H., Zhang, Y., Kong, D., Li, S., \& Yang, N. (2020). The effects of social support on sleep quality of medical staff treating patients with coronavirus disease 2019 (COVID-19) in January and February 2020 in China. Medical science monitor: international medical journal of experimental and clinical research, 26, e923549-1.

PeerJ reviewing PDF | (2021:02:58059:3:0:NEW 13 Apr 2021) 
441 37. Arafa, A., Mohammed, Z., Mahmoud, O., Elshazley, M., \& Ewis, A. (2021). Depressed, anxious, and 442 stressed: What have healthcare workers on the frontlines in Egypt and Saudi Arabia experienced 443 during the COVID-19 pandemic?. Journal of affective disorders, 278, 365-371.

444 38. Dunn, V. K., \& Sacco, W. P. (1989). Psychometric evaluation of the Geriatric Depression Scale and 445 the Zung Self-Rating Depression Scale using an elderly community sample. Psychology and $446 \quad$ Aging, 4(1), 125. 


\section{Table 1 (on next page)}

Table_1

Demographic profile of the respondents 
Table 1. Demographic profile of the respondents

\begin{tabular}{|c|c|c|}
\hline Demographic Profile & $n$ & $\%$ \\
\hline \multicolumn{3}{|c|}{ Type of health care facility } \\
\hline Government & 202 & 31.3 \\
\hline Private & 444 & 68.7 \\
\hline \multicolumn{3}{|l|}{ Age } \\
\hline 20-30 Years Old & 123 & 32.4 \\
\hline 31-40 Years Old & 177 & 46.6 \\
\hline 41-50 Years Old & 63 & 16.6 \\
\hline Above 50 Years Old & 17 & 4.5 \\
\hline \multicolumn{3}{|l|}{ Gender } \\
\hline Male & 167 & 26.0 \\
\hline Female & 475 & 74.0 \\
\hline \multicolumn{3}{|l|}{ Nationality } \\
\hline Saudi & 189 & 29.6 \\
\hline Non-Saudi & 450 & 70.4 \\
\hline \multicolumn{3}{|l|}{ Health specialty } \\
\hline Physicians & 114 & 17.7 \\
\hline Nurses & 323 & 50.2 \\
\hline Others & 207 & 32.1 \\
\hline \multicolumn{3}{|l|}{ Hospital Department } \\
\hline ER & 52 & 8.2 \\
\hline Wards & 134 & 21.1 \\
\hline ICU & 243 & 38.3 \\
\hline Others & 206 & 32.4 \\
\hline \multicolumn{3}{|l|}{ Years of experience } \\
\hline 1-5 Years & 280 & 44.3 \\
\hline $6-10$ Years & 177 & 28.0 \\
\hline 11 Years and Above & 175 & 27.7 \\
\hline \multicolumn{3}{|c|}{ Sleeping disorder before COVID-19 } \\
\hline Yes & 80 & 13.1 \\
\hline No & 532 & 86.9 \\
\hline \multicolumn{3}{|l|}{ Mental Disorder } \\
\hline Yes & 20 & 3.3 \\
\hline No & 592 & 96.7 \\
\hline
\end{tabular}

2

3

4 
Table 2 (on next page)

Table_2

Level of depression 
1

Table 2. Level of depression

\begin{tabular}{lcc}
\hline Level & Frequency & Percent \\
\hline Normal & 459 & 70.6 \\
Mild Depression & 170 & 26.2 \\
Moderate/Major & 16 & 2.5 \\
Severe/Extreme & 5 & 0.8 \\
Total & 650 & 100.0 \\
\hline
\end{tabular}

2

3 
Table 3 (on next page)

Table_3

The Association between demographic profile and Depression 
Table 3. The Association between demographic profile and Depression

\begin{tabular}{|c|c|c|c|c|c|c|c|c|c|c|c|}
\hline \multirow{2}{*}{ Variables } & \multicolumn{2}{|c|}{ Normal } & \multicolumn{2}{|c|}{ Mild } & \multicolumn{2}{|c|}{ Moderate } & \multicolumn{2}{|c|}{ Severe } & \multirow{2}{*}{ Value } & \multirow{2}{*}{$d f$} & \multirow{2}{*}{ sig. } \\
\hline & $\mathbf{N}$ & $\%$ & $\mathbf{N}$ & $\%$ & $\mathbf{n}$ & $\%$ & $n$ & $\%$ & & & \\
\hline \multicolumn{12}{|c|}{ Type of health care facility } \\
\hline Government & 152 & 75.2 & 40 & 19.8 & 9 & 4.5 & 1 & 0.5 & \multirow[t]{2}{*}{$2.377^{\mathrm{a}}$} & \multirow[t]{2}{*}{2} & \multirow[t]{2}{*}{0.305} \\
\hline Private & 306 & 68.9 & 127 & 28.6 & 7 & 1.6 & 4 & 0.9 & & & \\
\hline \multicolumn{9}{|l|}{ Age* } & \multirow{5}{*}{$12.892^{\mathrm{a}}$} & \multirow{5}{*}{6} & \multirow{5}{*}{0.045} \\
\hline $20-30$ & 94 & 76.4 & 25 & 20.3 & 4 & 3.3 & 4 & 2.3 & & & \\
\hline $31-40$ & 133 & 75.1 & 33 & 18.6 & 7 & 4.0 & 4 & 2.3 & & & \\
\hline $41-50$ & 53 & 84.1 & 9 & 14.3 & 1 & 1.6 & & & & & \\
\hline Above 50 years & 15 & 88.2 & 2 & 11.8 & & & & & & & \\
\hline \multicolumn{12}{|l|}{ Gender } \\
\hline Male & 129 & 77.2 & 35 & 21.0 & 1 & 0.6 & 2 & 1.2 & \multirow[t]{2}{*}{$3.683^{\mathrm{a}}$} & \multirow[t]{2}{*}{2} & \multirow[t]{2}{*}{0.159} \\
\hline Female & 329 & 69.3 & 128 & 26.9 & 15 & 3.2 & 3 & 0.6 & & & \\
\hline \multicolumn{12}{|l|}{ Nationality* } \\
\hline Saudi & 130 & 68.8 & 50 & 26.5 & 8 & 4.2 & 1 & 0.5 & \multirow{2}{*}{$8.321^{\mathrm{a}}$} & \multirow[t]{2}{*}{2} & \multirow{2}{*}{0.016} \\
\hline Non Saudi & 325 & 72.2 & 113 & 25.1 & 8 & 1.8 & 4 & 0.9 & & & \\
\hline \multicolumn{12}{|l|}{ Health specialty* } \\
\hline Physicians & 83 & 72.8 & 21 & 18.4 & 8 & 7.0 & 2 & 1.8 & \multirow{3}{*}{$9.543^{\mathrm{a}}$} & & \\
\hline Nurses & 237 & 73.4 & 78 & 24.1 & 5 & 1.5 & 3 & 0.9 & & 4 & 0.049 \\
\hline Others & 137 & 66.2 & 67 & 32.4 & 3 & 1.4 & & & & & \\
\hline Hospital Departmen & & & & & & & & & & & \\
\hline ER Department & 35 & 67.3 & 13 & 25.0 & 3 & 5.8 & 1 & 1.9 & & & \\
\hline Ward & 98 & 73.1 & 32 & 23.9 & 4 & 3.0 & & & $10.216^{\mathrm{a}}$ & 6 & 0.116 \\
\hline ICU Department & 173 & 71.2 & 58 & 23.9 & 8 & 3.3 & 4 & 1.6 & & & \\
\hline Others & 148 & 71.8 & 57 & 27.7 & 1 & 0.5 & & & & & \\
\hline Years of experience & & & & & & & & & & & \\
\hline 1-5Years & 206 & 73.6 & 68 & 24.3 & 5 & 1.8 & 1 & 0.4 & & & \\
\hline 6-10Years & 111 & 62.7 & 56 & 31.6 & 7 & 4.0 & 3 & 1.7 & $7.134^{\mathrm{a}}$ & 4 & 0.129 \\
\hline 11 Years and Above & 136 & 77.7 & 35 & 20.0 & 3 & 1.7 & 1 & 0.6 & & & \\
\hline $\begin{array}{l}\text { Sleeping disorder be } \\
\text { Covid-19* }\end{array}$ & & & & & & & & & & & \\
\hline Yes & 55 & 68.8 & 19 & 23.8 & 5 & 6.3 & 1 & 1.3 & $12.127^{\mathrm{a}}$ & 2 & 0.002 \\
\hline No & 394 & 74.1 & 123 & 23.1 & 11 & 2.1 & 4 & 0.8 & & & \\
\hline Mental Disorder & & & & & & & & & & & \\
\hline Yes & 10 & 50.0 & 8 & 40.0 & 2 & 10.0 & & & $5.919^{\mathrm{a}}$ & 2 & 0.052 \\
\hline No & 438 & 74.0 & 135 & 22.8 & 14 & 2.4 & 5 & 0.8 & & & \\
\hline
\end{tabular}




\section{Table 4 (on next page)}

Table_4

Descriptive analysis (frequency, percentage, mean and SD) 
Table 4. Descriptive analysis (frequency, percentage, mean and SD)

\begin{tabular}{|c|c|c|c|c|}
\hline Demographic Profile & $N$ & $\%$ & Mean & SD \\
\hline \multicolumn{5}{|c|}{ Type of health care facility } \\
\hline Government & 202 & 31.3 & 37.99 & 13.97 \\
\hline Private & 444 & 68.7 & 41.74 & 11.51 \\
\hline \multicolumn{5}{|l|}{ Age* } \\
\hline 20-30 Years Old & 123 & 32.4 & 38.12 & 12.79 \\
\hline 31-40 Years Old & 177 & 46.6 & 39.59 & 13.76 \\
\hline 41-50 Years Old & 63 & 16.6 & 36.98 & 11.07 \\
\hline Above 50 Years Old & 17 & 4.5 & 32.37 & 11.38 \\
\hline \multicolumn{5}{|l|}{ Gender } \\
\hline Male & 167 & 26.0 & 38.01 & 12.91 \\
\hline Female & 475 & 74.0 & 41.37 & 12.18 \\
\hline \multicolumn{5}{|l|}{ Nationality* } \\
\hline Saudi & 189 & 29.6 & 40.08 & 13.79 \\
\hline Non Saudi & 450 & 70.4 & 40.68 & 11.89 \\
\hline \multicolumn{5}{|l|}{ Health specialty* } \\
\hline Physicians & 114 & 17.7 & 39.70 & 14.30 \\
\hline Nurses & 323 & 50.2 & 40.09 & 12.10 \\
\hline Others & 207 & 32.1 & 41.70 & 11.85 \\
\hline \multicolumn{5}{|l|}{ Hospital Department } \\
\hline ER Department & 52 & 8.2 & 41.84 & 13.50 \\
\hline Ward & 134 & 21.1 & 40.06 & 11.58 \\
\hline ICU Department & 243 & 38.3 & 38.83 & 14.23 \\
\hline Others & 206 & 32.4 & 42.26 & 10.12 \\
\hline \multicolumn{5}{|l|}{ Years of experience } \\
\hline $1-5$ Years & 280 & 44.3 & 40.93 & 11.82 \\
\hline 6-10Years & 177 & 28.0 & 42.25 & 13.03 \\
\hline 11 Years and Above & 175 & 27.7 & 37.53 & 12.46 \\
\hline \multicolumn{5}{|c|}{ Sleeping disorder before } \\
\hline Yes & 80 & 13.1 & 41.20 & 15.18 \\
\hline No & 532 & 86.9 & 39.93 & 12.04 \\
\hline \multicolumn{5}{|l|}{ Mental Disorder } \\
\hline Yes & 20 & 3.3 & 43.91 & 15.27 \\
\hline No & 592 & 96.7 & 40.07 & 12.35 \\
\hline
\end{tabular}


Figure 1

Level of depression among study participants 


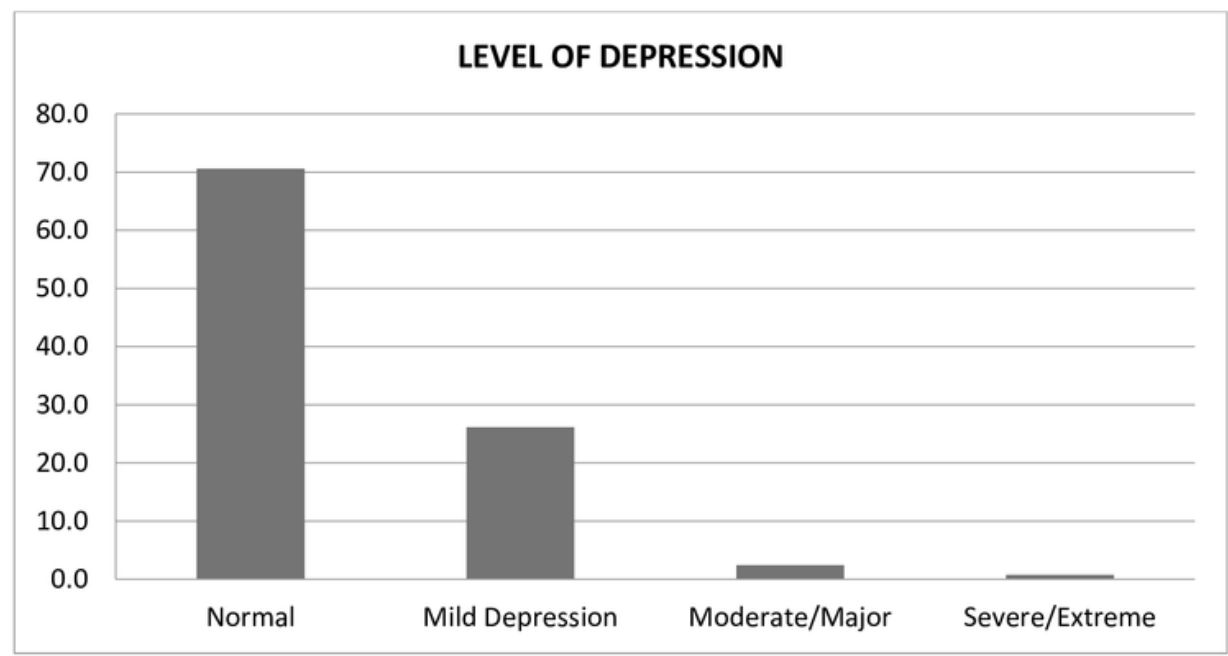

